Supporting Information

\title{
Incorporating Photochromic Triphenylamine into a Zirconium-Organic Framework for Highly Effective Photocatalytic Aerobic Oxidation of Sulfides
}

Xin-Nan Zou, ${ }^{\dagger}$ Deshan Zhang, ${ }^{\dagger}$ Tian-Xiang Luan, ${ }^{\dagger}$ Qiang Li, ${ }^{\dagger}$ Lei Li,${ }^{\dagger}$ Pei-Zhou Li, $,{ }^{*}, \dagger, *$ and Yanli Zhao*,

†School of Chemistry and Chemical Engineering, Shandong University, No.27 South Shanda Road, Jinan 250100, P. R. China

Division of Chemistry and Biological Chemistry, School of Physical and Mathematical Sciences, Nanyang Technological University, 21 Nanyang Link, 637371, Singapore

*Corresponding Authors. E-mail: pzli@sdu.edu.cn; zhaoyanli@ntu.edu.sg 


\section{Experimental section}

General information. All starting materials are commercially available and were used as received unless specifically mentioned.

Powder X-ray diffraction (PXRD) data were collected at $40 \mathrm{kV}, 200 \mathrm{~mA}$ on a SmartLab 9 $\mathrm{kW}$ X-ray diffractometer using $\mathrm{Cu}-\mathrm{K} \alpha$ radiation $\left(\lambda=1.5418 \AA\right.$ ) over $2 \theta$ range of $2.0^{\circ}-50.0^{\circ}$ at room temperature. Thermogravimetric analyses (TGA) were carried out on a TGA 5500 with a heating rate of $10{ }^{\circ} \mathrm{C} \mathrm{min}^{-1}$ under $\mathrm{N}_{2}$ flow $(60 \mathrm{~mL} / \mathrm{min})$ from room temperature to 650 ${ }^{\circ} \mathrm{C}$. Gas sorption analyses were conducted using Kubo-X1000 with extra-high pure gases. ${ }^{1} \mathrm{H}$ NMR spectra were measured on a Bruker AVANCE-400 spectrometer $\left(400 \mathrm{MHz}{ }^{1} \mathrm{H}\right)$ at ambient temperature. Transmission electron microscopy (TEM) images were recorded using a HT-7700 (100KV). The Electron paramagnetic resonance (EPR) spectra measurements were carried out on a JEOL RESONANCE (JES-X320) with Xe lamp (CEL-HXF300) as light source.

AFM measurements. The activated MOF sample was dispersed in ethanol solution firstly, and then an ultrasonication for $5 \mathrm{~min}$ followed by a static settlement process for $30 \mathrm{~min}$ were carried out. Finally, the upper layer solution was dropped by a pipette onto silicon substrate for atomic force microscopy (AFM) imaging. AFM measurements were performed on a Cypher ES AFM (Asylum Research, USA) in AC mode, and silicon cantilever (OMCL-AC240TS-R3, Olympus) with resonant frequency of $70 \mathrm{kHz}$ and spring constant of $2.0 \mathrm{~N} \mathrm{~m}^{-1}$ was employed. 

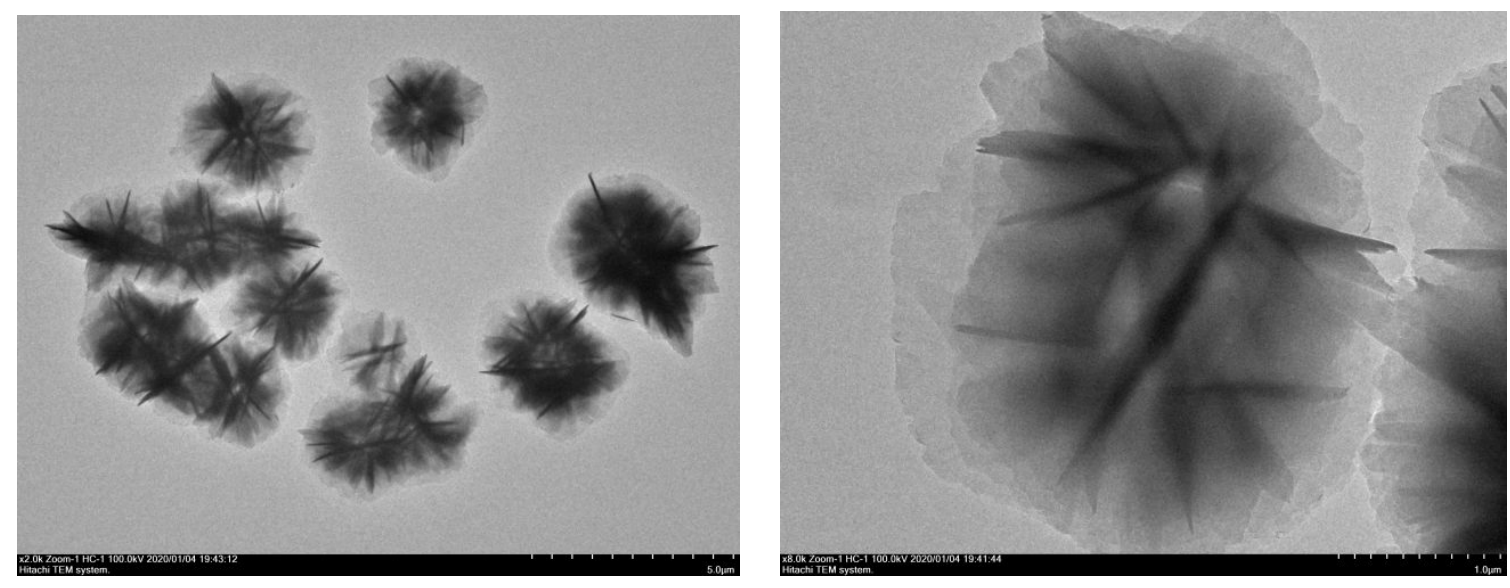

Figure S1. TEM images of synthesized $\mathbf{Z r}_{12}-\mathbf{N B C}$.
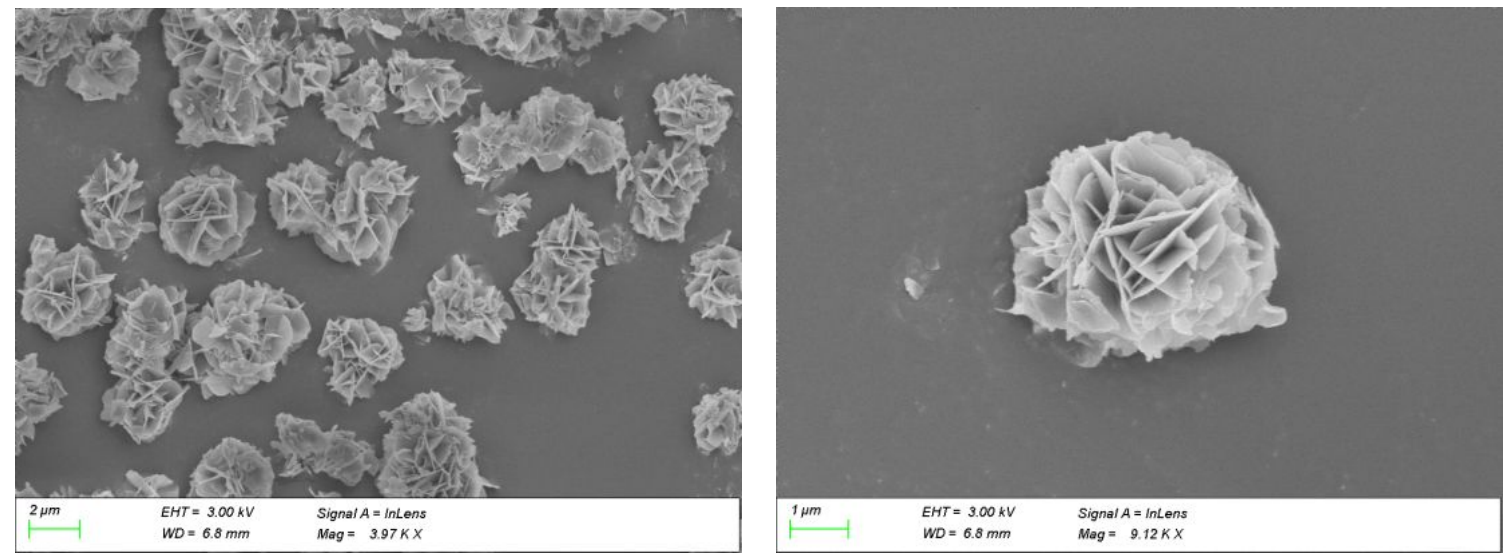

Figure S2. SEM images of synthesized $\mathbf{Z} \mathbf{r}_{12}-\mathbf{N B C}$. 


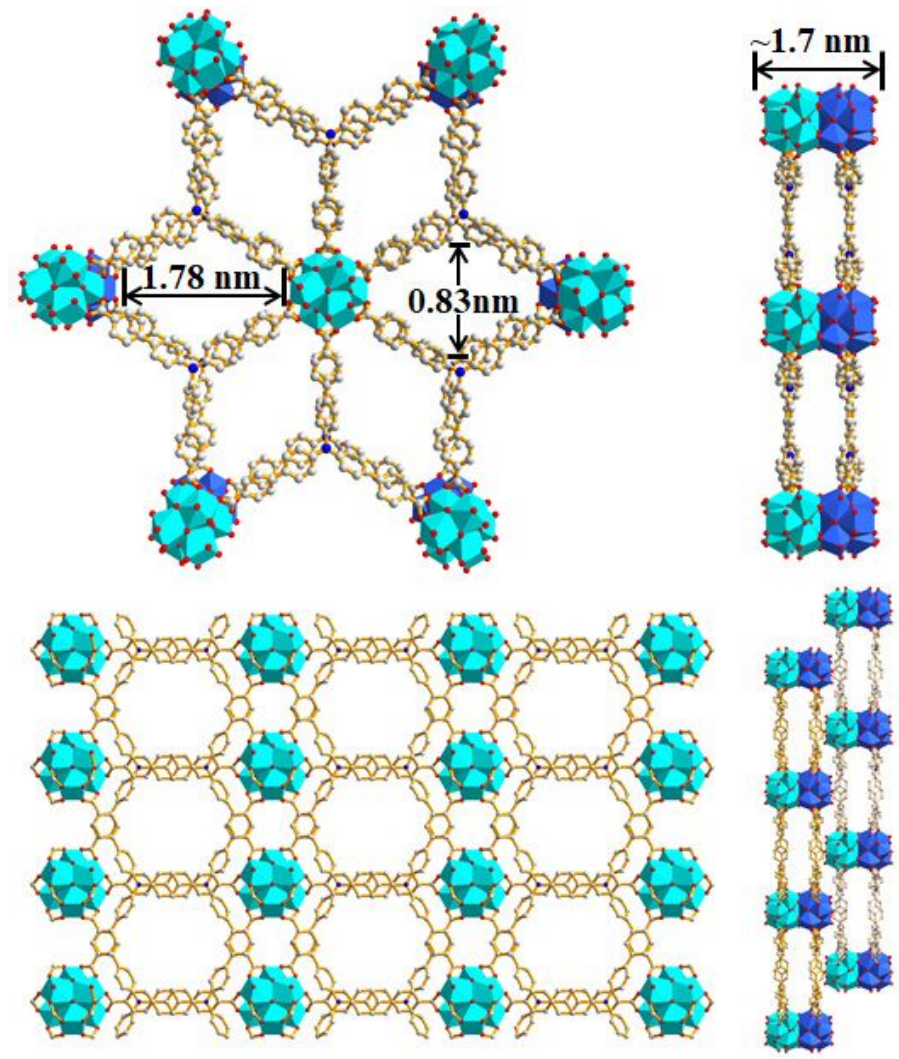

Figure S3. Simulated crystal structure of synthesized $\mathbf{Z r}_{\mathbf{1 2}}-\mathbf{N B C}$.

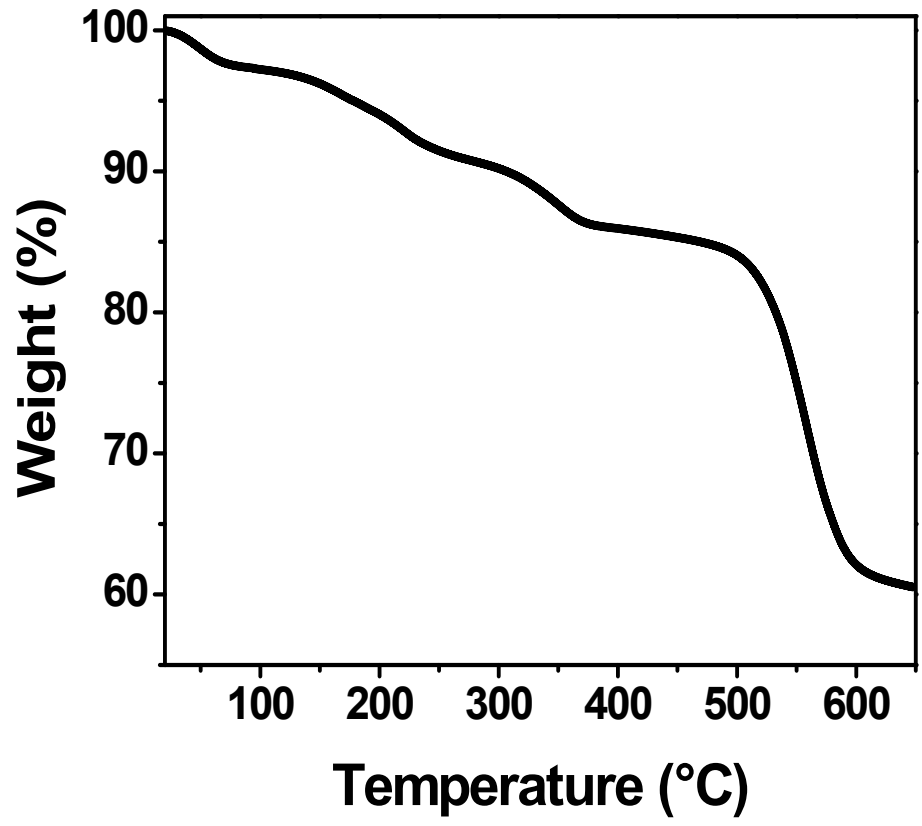

Figure S4. TGA curve of activated $\mathbf{Z r}_{12}-\mathbf{N B C}$, washed by ethanol and acetone and dried at $150{ }^{\circ} \mathrm{C}$ for 6 hours. 


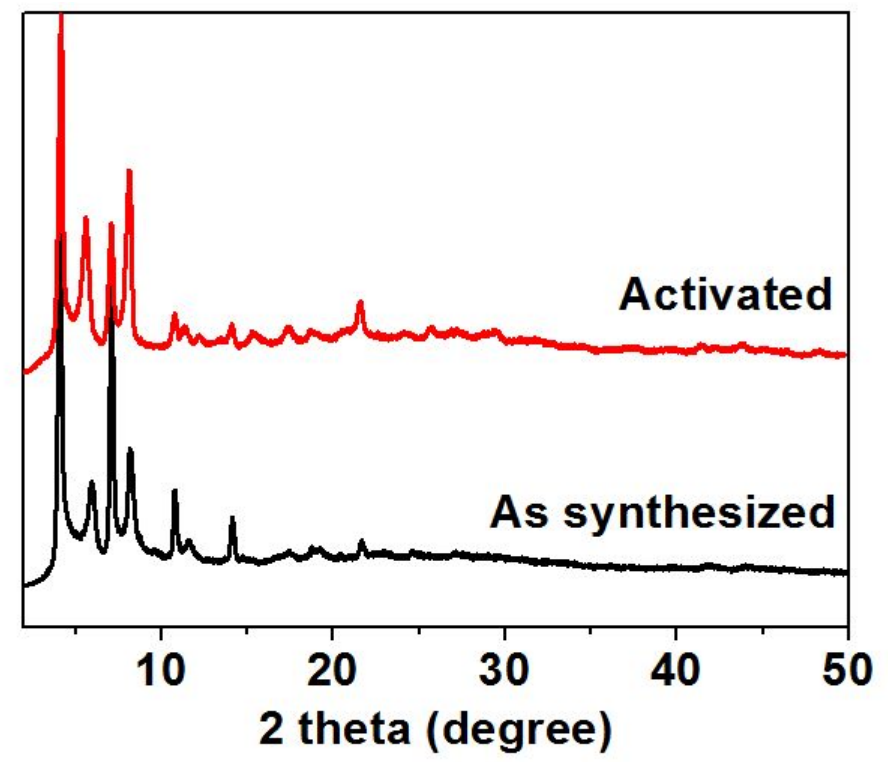

Figure S5. Powder X-ray diffraction patterns of as-synthesized and activated $\mathbf{Z r}_{\mathbf{1 2}}-\mathbf{N B C}$.
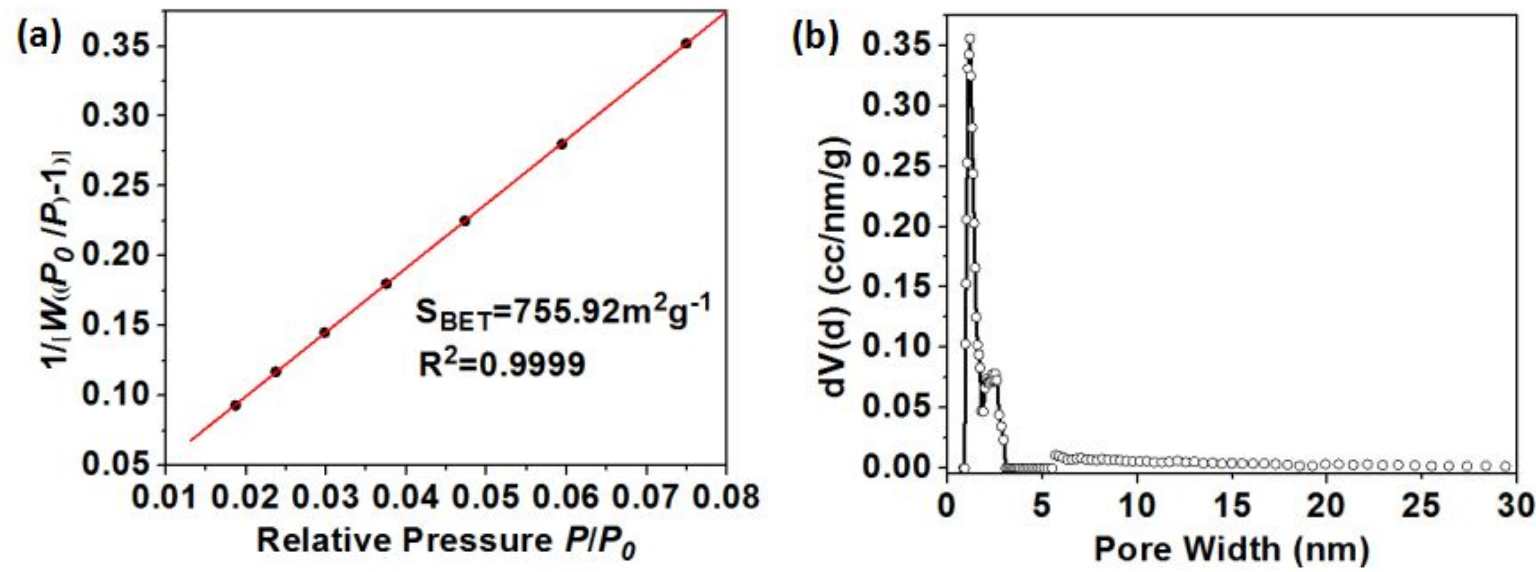

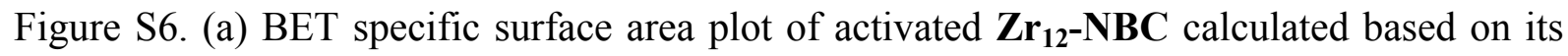
$\mathrm{N}_{2}$ adsorption isotherm at $77 \mathrm{~K}$. (b) Pore-size distribution profile of $\mathbf{Z r}_{\mathbf{1 2}}-\mathbf{N B C}$. 

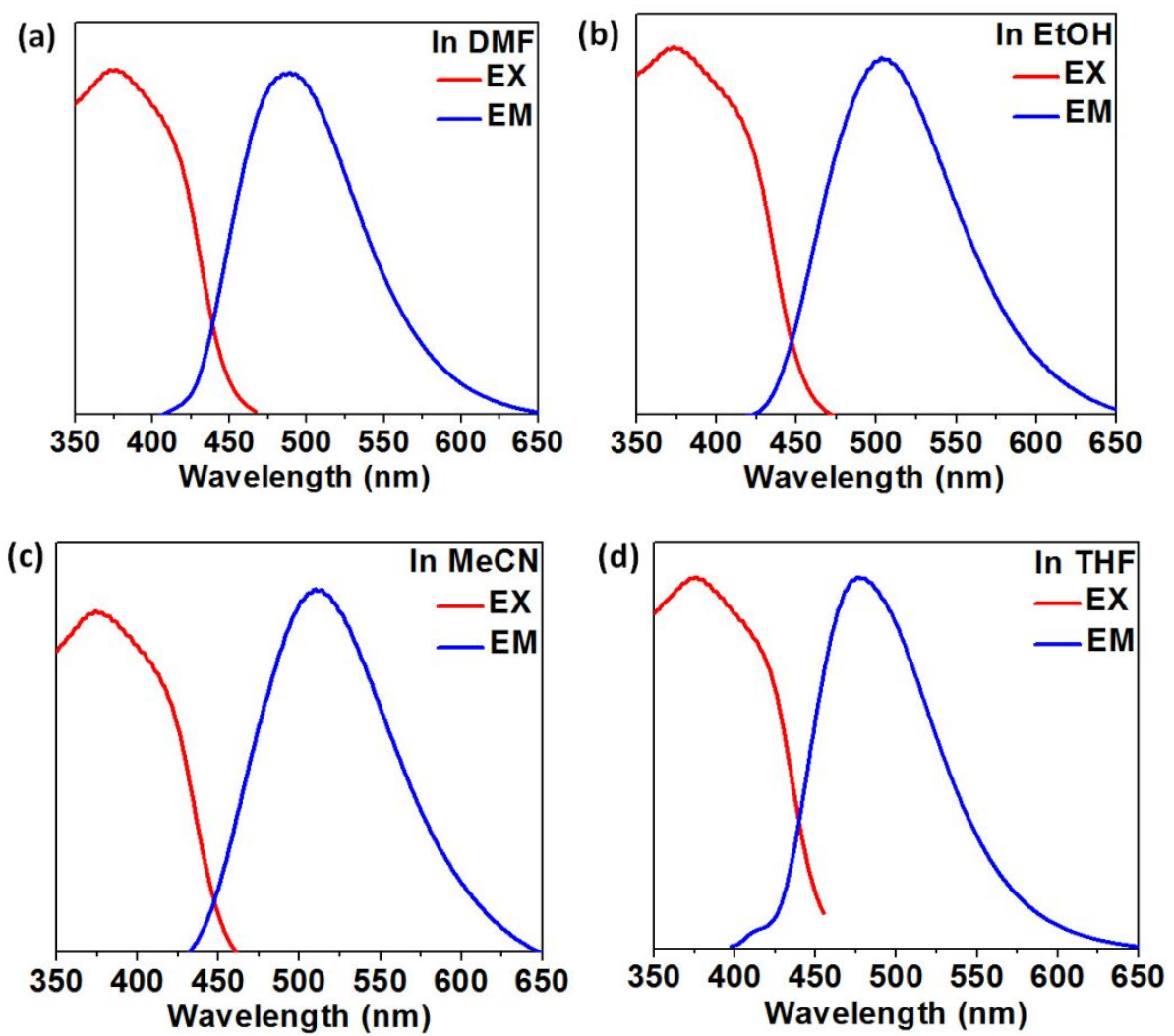

Figure S7. Fluorescence spectra of $\mathbf{Z r}_{\mathbf{1 2}}-\mathbf{N B C}$ in (a) DMF, (b) EtOH, (c) MeCN, and (d) THF.

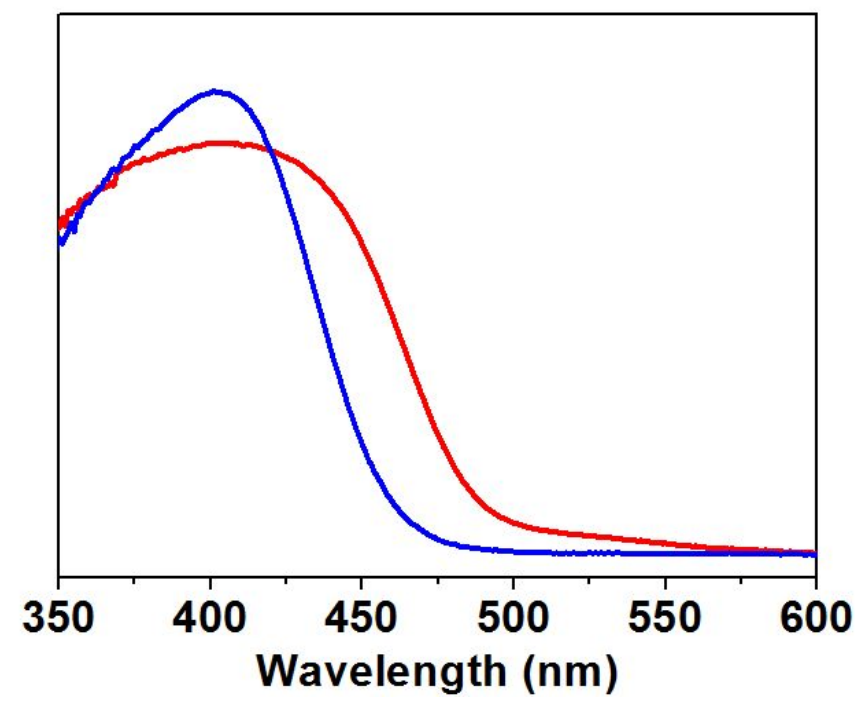

Figure S8. UV-Vis spectra of $\mathbf{Z r}_{\mathbf{1 2}}$-NBC (blue) and $\mathrm{H}_{3} \mathrm{NBC}$ (red) in the solid state. 


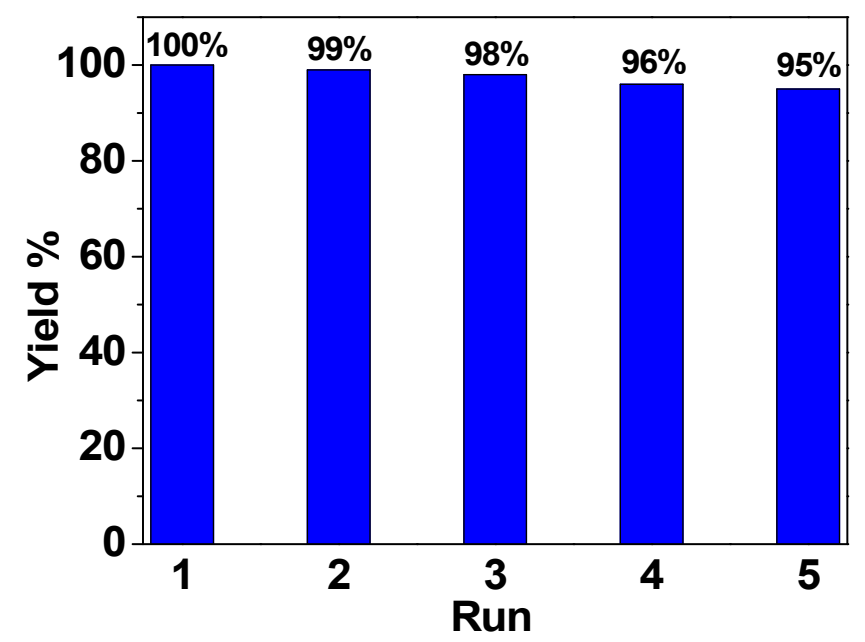

Figure S9. Photocatalytic activity of $\mathbf{Z} \mathbf{r}_{\mathbf{1 2}} \mathbf{- N B C}$ on aerobic oxidation of thioanisole over five cycles.

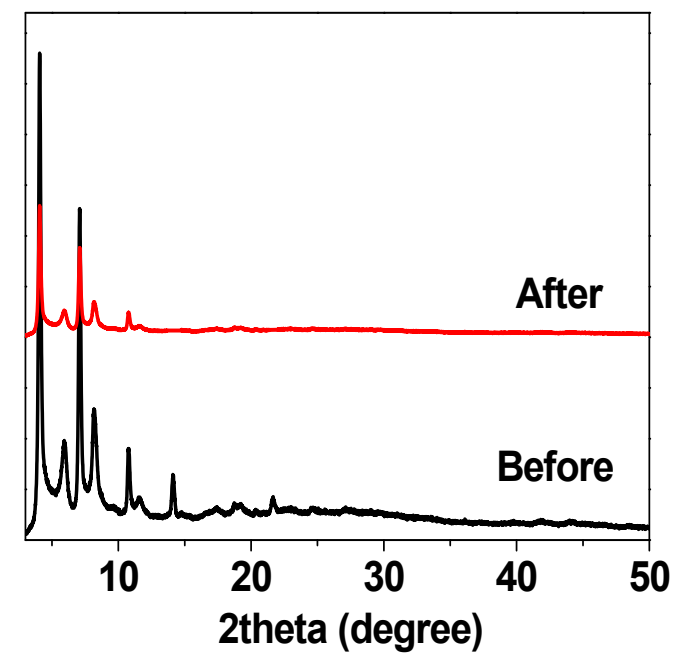

Figure S10. Powder X-ray diffraction patterns of $\mathbf{Z r}_{\mathbf{1 2}^{2}}-\mathbf{N B C}$ before (black line) and after (red line) photocatalytic reaction. 


\section{Characterization of the photocatalytic products}

\section{(Methylsulfinyl)benzene}<smiles>CS(=O)c1ccccc1</smiles>

The title compound was made following the general procedure using thioanisole ( $35 \mu \mathrm{L}, 0.30$ $\mathrm{mmol})$ as the starting material. The product was obtained as a colorless liquid (100\% yield). ${ }^{1} \mathrm{H}$ NMR (400 MHz, MeOD): $\delta 7.69(\mathrm{~d}, \mathrm{~J}=1.6 \mathrm{~Hz}, 3 \mathrm{H}), 7.62-7.55(\mathrm{~m}, 2 \mathrm{H}), 2.79(\mathrm{~s}, 3 \mathrm{H})$.

\section{1-Fluoro-4-(methylsulfinyl)benzene}<smiles>CS(=O)c1ccc(F)cc1</smiles>

The titled compound was made following the general procedure using 4-fluorothioanisole (36 $\mu \mathrm{L}, 0.30 \mathrm{mmol})$ as starting material. The product was obtained as a colorless liquid $(100 \%$ yield). ${ }^{1} \mathrm{H}$ NMR (400 MHz, MeOD): $\delta 7.81(\mathrm{~d}, \mathrm{~J}=5.1 \mathrm{~Hz}, 2 \mathrm{H}), 7.41(\mathrm{~d}, \mathrm{~J}=8.8 \mathrm{~Hz}, 2 \mathrm{H}), 2.85$ $(\mathrm{s}, 3 \mathrm{H})$.

\section{1-Chloro-4-(methylsulfinyl)benzene}<smiles>CS(=O)c1ccc(Cl)cc1</smiles>

The titled compound was made following the general procedure using (4-chlorophenyl)-(methyl)sulfane $(39 \mu \mathrm{L}, 0.30 \mathrm{mmol})$ as starting material. The product was obtained as a colorless liquid (100\% yield). ${ }^{1} \mathrm{H}$ NMR (400 MHz, MeOD): $\delta 7.70$ (d, J = 8.6 $\mathrm{Hz}, 2 \mathrm{H}), 7.61(\mathrm{~d}, \mathrm{~J}=8.6 \mathrm{~Hz}, 1 \mathrm{H}), 2.79(\mathrm{~s}, 3 \mathrm{H})$. 


\section{1-Bromo-4-methylsulfinylbenzene}<smiles>CS(=O)c1ccc(Br)cc1</smiles>

The titled compound was made following the general procedure using 4-bromothioanisole (61 $\mathrm{mg}, 0.30 \mathrm{mmol})$ as starting material. The product was obtained as a colorless liquid $(77 \%$ yield). ${ }^{1} \mathrm{H}$ NMR (400 MHz, MeOD): $\delta 7.83(\mathrm{~d}, \mathrm{~J}=11.6 \mathrm{~Hz}, 2 \mathrm{H}), 7.62(\mathrm{~d}, \mathrm{~J}=8.7 \mathrm{~Hz}, 2 \mathrm{H})$, $2.79(\mathrm{~s}, 3 \mathrm{H})$.

\section{1-Methyl-4-(methylsulfinyl)benzene}<smiles>Cc1ccc(S(C)=O)cc1</smiles>

The title compound was made following the general procedure using methyl(p-tolyl)sulfane (40 $\mu \mathrm{L}, 0.30 \mathrm{mmol}$ ) as starting material. The product was obtained as a colorless liquid (100\% yield). ${ }^{1} \mathrm{H}$ NMR (400 MHz, MeOD): $\delta 7.65$ (d, J = 8.3 Hz, 2H), 7.47 (d, J = 7.9 Hz, 2H), 2.83 $(\mathrm{s}, 3 \mathrm{H}), 2.48(\mathrm{~s}, 3 \mathrm{H})$.

\section{1-Methanesulfinyl-4-methoxybenzene}<smiles>COc1ccc(S(C)=O)cc1</smiles>

The title compound was made following the general procedure using $p$-methylthioanisole (46 $\mathrm{mg}, 0.25 \mathrm{mmol})$ as starting material. The product was obtained as a colorless liquid $(80 \%$ yield). ${ }^{1} \mathrm{H}$ NMR (400 MHz, MeOD): $\delta 7.68$ (d, J = 9.0 Hz, 2H), 7.14 (s, 2H), 3.88 (s, 3H), $2.79(\mathrm{~s}, 3 \mathrm{H})$. 


\section{2-Methylsulfinylnaphthalene}<smiles>CS(=O)c1ccc2ccccc2c1</smiles>

The title compound was made following the general procedure using 2-(methylthio)-naphthalene $(52 \mathrm{mg}, 0.30 \mathrm{mmol})$ as starting material. The product was obtained as a colorless liquid (75\% yield). ${ }^{1} \mathrm{H}$ NMR (400 MHz, MeOD): $\delta 8.30$ (s, 1H), 8.15 $(\mathrm{d}, \mathrm{J}=8.7 \mathrm{~Hz}, 2 \mathrm{H}), 7.79(\mathrm{dd}, \mathrm{J}=8.7,1.8 \mathrm{~Hz}, 1 \mathrm{H}), 7.70-7.66(\mathrm{~m}, 2 \mathrm{H}), 2.93(\mathrm{~s}, 3 \mathrm{H})$.

\section{Ethylsulfinylbenzene}<smiles>CCS(=O)c1ccccc1</smiles>

The title compound was made following the general procedure using phenylethylthiol $(41 \mu \mathrm{L}$, $0.30 \mathrm{mmol}$ ) as starting material. The product was obtained as a colorless liquid (91\% yield). ${ }^{1} \mathrm{H}$ NMR (400 MHz, MeOD): $\delta 7.71(\mathrm{~d}, \mathrm{~J}=1.7 \mathrm{~Hz}, 2 \mathrm{H}), 7.63$ (d, J = 1.7 Hz, 3H), 3.06 (dd, J $=11.3,3.8 \mathrm{~Hz}, 2 \mathrm{H}), 1.22(\mathrm{~s}, 3 \mathrm{H})$.

\section{p-Tolyl ethyl sulfoxide}<smiles>CCS(=O)c1ccc(C)cc1</smiles>

The title compound was made following the general procedure using 1-ethysulfanyl-4-methylbenzene $(46 \mu \mathrm{L}, 0.30 \mathrm{mmol}$ ) as starting material. The product was obtained as a colorless liquid (89\% yield). ${ }^{1} \mathrm{H}$ NMR (400 MHz, MeOD): $\delta 7.60$ (d, J = 8.2 Hz, 2H), 7.47 (d, J = 8.4 Hz, 2H), $3.04(\mathrm{dd}, \mathrm{J}=14.1,6.8 \mathrm{~Hz}, 2 \mathrm{H}), 2.48(\mathrm{~s}, 3 \mathrm{H}), 1.21(\mathrm{~s}, 3 \mathrm{H})$. 


\section{1-(Benzenesulfinyl)-4-methylbenzene}<smiles>Cc1ccc(S(=O)c2ccccc2)cc1</smiles>

The title compound was made following the general procedure using 4-methylphenyl phenyl sulfide (54 $\mu \mathrm{L}, 0.30 \mathrm{mmol})$ as starting material. The product was obtained as a colorless liquid (81\% yield). ${ }^{1} \mathrm{H}$ NMR (400 MHz, MeOD): $\delta 7.32(\mathrm{~d}, \mathrm{~J}=2.1 \mathrm{~Hz}, 2 \mathrm{H}), 7.31-7.24(\mathrm{~m}, 5 \mathrm{H}), 7.22$ $(\mathrm{d}, \mathrm{J}=7.9 \mathrm{~Hz}, 2 \mathrm{H}), 2.38(\mathrm{~s}, 3 \mathrm{H})$.

\section{1-Chloro-2-ethylsulfinylethane}<smiles>CCS(=O)CCCl</smiles>

The title compound was made following the general procedure using 2-chloroethyl ethyl sulfide $(35 \mu \mathrm{L}, 0.30 \mathrm{mmol})$ as starting material. The product was obtained as a colorless liquid (85\% yield). ${ }^{1} \mathrm{H}$ NMR (400 MHz, MeOD): $\delta 3.66(\mathrm{q}, \mathrm{J}=7.1 \mathrm{~Hz}, 2 \mathrm{H}), 2.74(\mathrm{t}, \mathrm{J}=6.7 \mathrm{~Hz}, 2 \mathrm{H})$, 2.62 (q, J = 7.4 Hz, 2H), 1.29 (t, J = 7.4 Hz, 3H). 